\title{
Application of Var Method
}

\author{
Ping $\mathrm{Hu}^{1,2 \mathrm{a}}$ and Jinwu Fang ${ }^{3 \mathrm{~b}}$ \\ 1College of mathematics \& computer Wuhan Textile University Wuhan ,430073, China \\ 2College of economics Economics and Law of Zhongnan university Wuhan ,430073, China \\ 3 School of Economics Shanghai University Shanghai, 200041, China \\ amay2001@wtu.edu.cn, bangjinwu007@126.com
}

Keywords: Insurance; Risk factor; Var; Co-variance method; Multivariate normal distribution

\begin{abstract}
Since the 2008 financial crisis, the European debt crisis continuous fermentation in the background, the financial risk has become the focus concern of governments. Risk measurement is the core part of risk management in China, the opening of financial market background, research on risk measurement is of very important significance. A global perspective, the main method of risk measurement is Var in the insurance market. This paper introduces the application of Var in insurance, calculate and analyze their respective Var
\end{abstract}

\section{Introduction (Heading 1)}

The foundation and the core of risk management is the quantitative analysis and assessment of risk. With the increase of the financial market and financial transaction scale, dynamics and complexity, the development of financial theory and financial engineering, risk measurement technology has become more sophisticated, comprehensive.

The main financial market risk measurement methods, according to the time order of sensitivity analysis, fluctuation analysis method, Var, stress testing and extreme value analysis and other methods, each method can solve a class of problems. This paper mainly researches the application of Var in insurance.

At present, unable to find a suitable method to measure the risk in all cases in the world. The risk of individual securities analysis measure for volatility, this simple, easy operation so that each investor can learn. Sensitivity analysis is a kind of auxiliary method, because it requires a linear relationship between the rate of return and risk, this is unrealistic. Var Method is the most popular global risk measure method, but at home, this method is beginning to spread. Var Method has its fatal weakness, under normal circumstances, risk of financial industry place is huge, at the extreme end, because the thick tail, not to capture this risk, so the introduction of a stress testing and extreme value theory to make up Var method.

\section{Introduction of Var}

The Definition. Given $\alpha \in[0,1], \quad \operatorname{VaR}_{\alpha}$ of stochastic risk $\mathrm{X}$ having probability distribution $\mathrm{p}$ in the confidence level $\alpha$ which is defined as: $\operatorname{Va}_{\alpha}=\inf \{x \in R \mid P(X \leq x) \geq \alpha\}$ This is usually defined. VaR depends on the certain time interval and the loss probability of two parameters. In a given time interval, if known the probability distribution function of random variables, the parameter expressions of $\operatorname{VaR}_{\alpha}$ can be calculated.

Estimation method with variance co-variance method, historical simulation method and Monte Carlo simulation method, this section mainly uses the variance - co-variance method in Delta- normal method[3] . Delta- normal method assumes that market factors change with multivariate normal distribution.we can calculate Var. 
Application in Insurance of Var. Var can be used in the insurance company's balance risk management, as an information disclosure tool of an insurance company, as an investment performance evaluation tool, but also can be used for insurance supervision, the internal model of Basel Committee selected interval of 10 days, $99 \%$ confidence interval, the calculated value is multiplied by a safety factor coefficient $(=3)$, it is guaranteed the minimum capital requirements for regulatory purposes. Insurance regulators can learn from the Basel Committee's proposal to require insurance companies to provide a certain period Var reports, using the quantitative criteria to determine the capital.

The method of risk management is the direction of development of the insurance industry, especially in the insurance funds in the future deeply involved in financial derivatives. China's securities market management is not perfect, in the transition state of non-market and market, currently in the domestic insurance companies and other companies are not universal, but in view of the current development situation, popular risk measure method is represent the general trend.

In a word, the Var method will produce significant influence in the insurance industry and the regulatory solvency[4], specifically, are: firstly, it will encourage some weak insurance companies limit the risk or increase of capital; secondly, it can encourage regulatory departments take corrective measures in the case as soon as possible; lastly, it can help regulators and other aspects identify those too little capital unable to achieve a lower level of risk of insolvent insurance companies.

\section{The Empirical Analysis}

Sample Selection. there are four companies of Shanghai A shares in the insurance plate: NCI, China Ping An , China Life and China Pacific insurance. In the big wisdom chose the daily data from March 22, 2012 to March 22, 2013 of the four companies, Assume that investors have $1000000 \mathrm{cash}$, the money invested the four insurance companies, if investment accounted for $25 \%$.

Results. Take "China Pacific Insurance" as Code 1, "China Life" as code 2, "China Ping An" as code 3, "NCI" as code 4. Use EXCEL to calculate the standard deviation, correlation matrix, co-variance matrix is as follows:

$$
\begin{aligned}
& \sigma_{1}=0.02120, \sigma_{2}=0.01935 \\
& \sigma_{3}=0.02022, \sigma_{4}=0.02936
\end{aligned}
$$

So we can get these correlation coefficient matrix:

$$
\text { coefficientMatrix }=\left(\begin{array}{cccc}
1 & 0.781054 & 0.704844 & 0.717989 \\
0.781054 & 1 & 0.672284 & 0.65396 \\
0.704844 & 0.672284 & 1 & 0.77963 \\
0.717989 & 0.65396 & 0.77963 & 1
\end{array}\right)
$$

Then we can get these co-variance matrix:

$$
c=\left[\begin{array}{cccc}
0.000858 & 0.000461 & 0.00037 & 0.000445 \\
0.000461 & 0.000407 & 0.000262 & 0.000301 \\
0.00037 & 0.000262 & 0.000373 & 0.000319 \\
0.000445 & 0.000301 & 0.000319 & 0.000447
\end{array}\right]
$$

Model Application. Take time t for 25 days, confidence level for the 95\%, considering the single risk factor and multiple risk factors.

- The word

- Single risk factor $\sigma$ normal-Var 
Without considering the correlation of four kinds of assets, according to the formula (2), can find out maximum possible adverse movements in interest rates in $95 \%$ confidence level of 25 days, so we can get all single factor Var and total Var:

$$
\operatorname{Var}=p_{0} * \varpi_{i} * \Delta I=p_{0} * \varpi_{i} * \Phi^{-1}(\alpha) * \sigma * \sqrt{\Delta t}
$$

Single Var:

$$
\begin{aligned}
& \operatorname{Var}_{1}=100 * 0.25 * 1.65 * 0.02120 * \sqrt{25}=4.3725 \\
& \operatorname{Var}_{2}=100 * 0.25 * 1.65 * 0.01935 * \sqrt{25}=3.9910 \\
& \operatorname{Var}_{3}=100 * 0.25 * 1.65 * 0.02022 * \sqrt{25}=4.1705 \\
& \operatorname{Var}_{4}=100 * 0.25 * 1.65 * 0.02936 * \sqrt{25}=6.0555
\end{aligned}
$$

Total Var:

Var $=18.5895$

- Multiple risk factors $\sigma$ normal-Var

In consideration of the asset correlation case, according to the formula (3),

$$
\operatorname{Var}_{\text {total }}=\sqrt{V * C * V^{T}}
$$

among them:

$$
\mathrm{V}=\left(\begin{array}{llll}
4.3725 & 3.9910 & 4.1705 & 6.0555
\end{array}\right)
$$

$\mathrm{C}$ is given by equation (1).So :

$$
\text { Var }_{\text {total }}=16.53763
$$

Can be seen that sum of the single risk factor Delta-Var normal than multiple risk factors to high, showing that diversification of assets and asset correlations reduce risk. In addition, the risk perspective, investors choose the best Chinese life.

At present, in the global scope, the use of Var in insurance company has become the mainstream, for the thick tail problems can not be solved by Var, 错误!未找到引用源。, theory analysis, stress testing and extreme value of the supplement have been put forward[6][7]. However, Var method has been able to resolve most of the insurance risk management

\section{Conclusions}

The foundation and the core of risk management is the quantitative analysis and assessment of risk. With the increase of the financial market and financial transaction scale, dynamics and complexity, the development of financial theory and financial engineering, risk measurement technology has become more sophisticated, comprehensive.

At present, unable to find a suitable method to measure the risk in all cases in the world. The risk of individual securities analysis measure for volatility, this simple, easy operation so that each investor can learn. Sensitivity analysis is a kind of auxiliary method, because it requires a linear relationship between the rate of return and risk, this is unrealistic. Var Method is the most popular global risk measure method, but at home, this method is beginning to spread. Var Method has its fatal weakness, under normal circumstances, risk of financial industry place is huge, at the extreme end, because the thick tail, not to capture this risk, so the introduction of a stress testing and extreme value theory to make up Var Method. 


\section{References}

[1] Chinese society of Actuaries group, Non-life insurance actuarial [M] China financial and Economic Publishing House, 2010.

[2] Liu jiaming, Method and Application Study on the financial risk measurement[D], Chongqing University, 2009

[3] Fu lili, the risk of the use of insurance funds in the measurement of [J], Financial, 2007, (11):77-79.

[4] Lin yuan, Lin xiao, Var method and its application in the risk management of insurance of our country[J] Journal of China Insurance Management Cadre Institute, 2002, (5): 42-44.

[5] Huang yingjun, the use of insurance funds in China risk theory research of [J] Journal of Yunnan University of Finance and Economics, 2010, (3):94-102.

[6] Zhong chun, $\mathrm{Wu}$ xue, Chen wenchai, and its application in the insurance fund investment portfolio optimization model based on Var and CVar [J]., Journal of Nanchang University, 2011, (33): 308-408.

[7] Siddharth Rajan Alexander. CVar and Var for a portfolio of derivatives [D]. America, New York: Cornell University. 